\title{
Endovascular therapy of symptomatic high-grade stenosis of left internal carotid artery in C6 segment using Elutax “3” Neuro pDEB
}

\author{
Paweł Latacz ${ }^{1}$, Tadeusz Popiela², Paweł Brzegowy ${ }^{2}$, Borys Kwinta ${ }^{3}$, Maciej Chwała ${ }^{4}$, Marian Simka \\ ${ }^{1}$ Department of Neurology, Jagiellonian University Medical College, University Hospital, Krakow, Poland \\ ${ }^{2}$ Chair of Radiology, Jagiellonian University Medical College, Krakow, Poland \\ ${ }^{3}$ Department of Neurosurgery and Neurotraumatology, Jagiellonian University Medical College, Krakow, Poland \\ ${ }^{4}$ Department of Surgery, Faculty of Health Sciences, Jagiellonian University Medical College, Krakow, Poland \\ ${ }^{5}$ Department of Anatomy, University of Opole, Opole, Poland
}

Adv Interv Cardiol 2021; 17, 3 (65): 332-333

DOI: https://doi.org/10.5114/aic.2021.109162

Intracranial atherosclerotic disease (ICAD) is a wellknown cause of stroke and is responsible for approximately $5-10 \%$ of all strokes [1]. The annual risk of recurrent stroke in symptomatic ICAD is around 9-12\% despite optimal medical treatment [2]. Patients presenting with symptomatic ICAD have been managed endovascularly (ET) for over two decades. Still, although initial results of such treatment were encouraging, the rates of periprocedural complications and restenoses were high, $15 \%$ and $34 \%$, respectively [2].

Recently, in order to improve the results of ET, novel methods such as drug-coated balloons (DEBs) are increasingly used in these patients. The DEBs are routinely used for the treatment of coronary artery disease, as well as in patients presenting with peripheral arterial lesions. Intracranial arteries (IA) are a new target for this endovascular tool. Since IA differ from the coronary ones and those of the extremities, in terms of their morphology, there are some devices registered for this unique application. The Elutax "3" Neuro drug coated balloon (AR Baltic Medical, Vilnius, Lithuania), which is a hydrophilic balloon covered with paclitaxel trapped in a dextran matrix, is one such device specifically designed for neurovascular applications. Of note, according to the manufacturer, this balloon does not require predilation, since the loss of its unique resistant polymer during the navigation through lesions is not higher than $5 \%$. The balloons are available on a rapid exchange catheter, diameter 1.5-6.0 mm and length 10-40 $\mathrm{mm}$.

In this report we present a case of ET in a 57-year-old patient presenting with stroke resulting from atheroscle- rotic stenosis in the $\mathrm{C} 5 / \mathrm{C} 6$ (clinoid/ophthalmic) segment of the internal carotid artery (ICA), who was managed with this specific endovascular device (first in Poland).

This patient presented with recurrent stroke of the left cerebral hemisphere. Angiography revealed a short critical stenosis in the $\mathrm{C5} / \mathrm{C} 6$ segment of the left ICA (Figure $1 \mathrm{~A}$ ) and also $60 \%$ stenosis in the $\mathrm{C} 5$ segment of the right ICA. Furthermore, there was no adequate collateral inflow to the left cerebral hemisphere from the right side.

Considering the previous history of this patient and angioarchitecture of his IA circle, we decided to address the lesion of the left ICA, endovascularly, using DEB and a proximal protection system. After introduction of the Mo.Ma 8F (Medtronic, Minneapolis, MA, USA) protection system, a Transcend wire (Boston Scientific, Natick, MA, USA) was navigated into the periphery of the left middle cerebral artery. One inflation of the $3.5 \times 15 \mathrm{~mm}$ Elutax 3 Neuro balloon, inflated under the pressure of $6 \mathrm{~atm}$ for $30 \mathrm{~s}$, was performed (Figure $1 \mathrm{~B}$ ). Of note, the duration of the balloon inflation, in comparison with extracranial arteries, was relatively short. Still, the producer of this particular balloon recommends a $15 \mathrm{~s}$ inflation. Considering the characteristics of the lesion, we performed a longer inflation, yet the $30 \mathrm{~s}$ time also included a slow and gentle filling of the balloon. The final angiographic result of the procedure was good (Figure $1 \mathrm{C}$ ). The postprocedural course of this patient was uneventful. He was discharged home with a recommendation to use dual antiplatelet platelet therapy (DAPT) up to 6 months after the procedure. During the 6-month follow-up, the patient did not develop any new neurological symptoms, and the

\section{Corresponding author:}

Paweł Latacz MD, PhD, Department of Neurology, Jagiellonian University Medical College, University Hospital, 2 Jakubowskiego St, 30-688 Krakow, Poland, phone: +48 1240025 51, e-mail: pawlat@me.com

Received: 24.05.2021, accepted: 7.08.2021. 

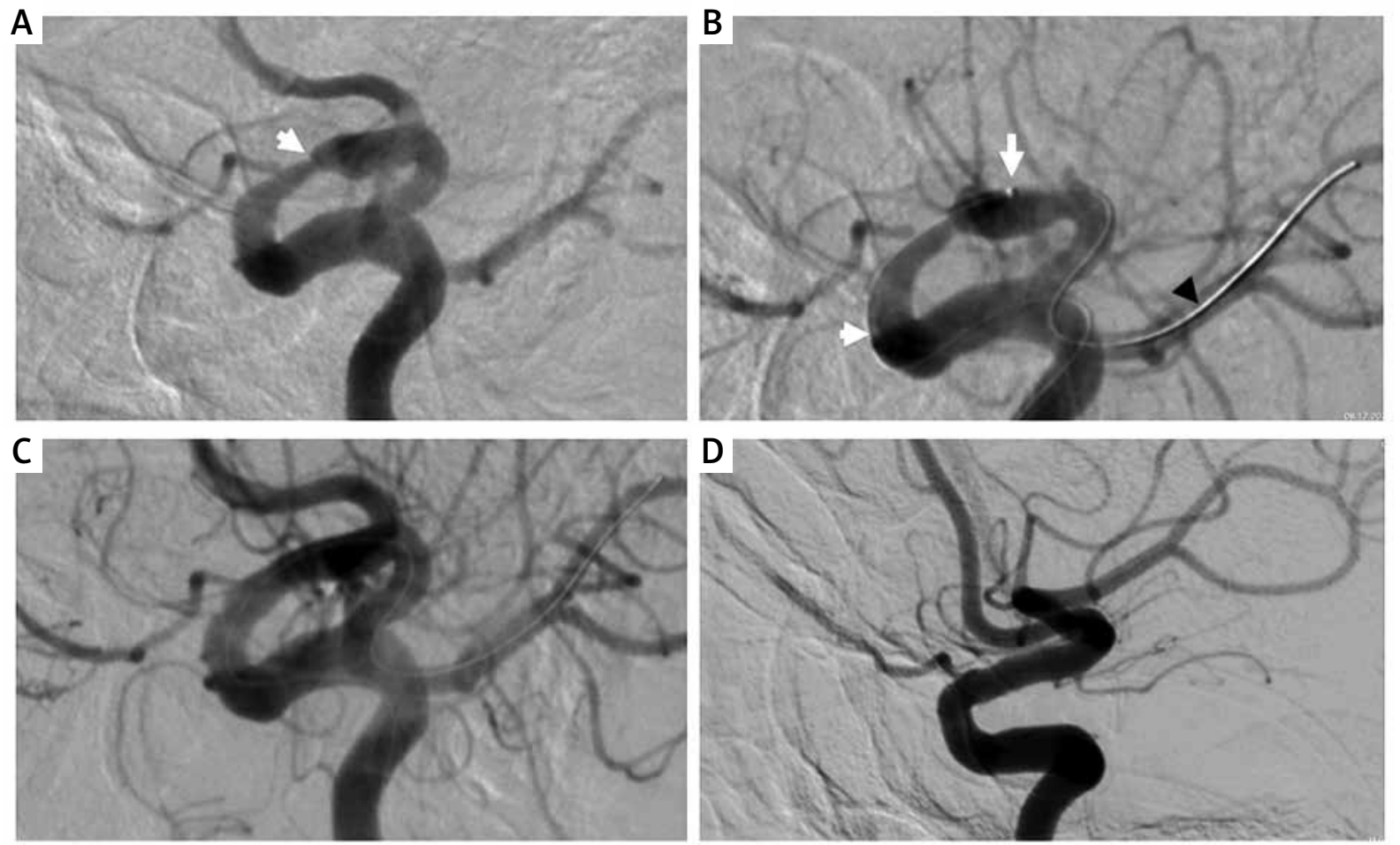

Figure 1. A - Critical stenosis of the left internal carotid artery in the C5/C6 segments (arrow), B - Elutax "3" Neuro drug coated balloon angioplasty at the site of the stenosis (balloon between white arrows, black arrow - guidewire in the middle cerebral artery), C - final result of angioplasty, $\mathbf{D}$ - follow-up angiography after 6 months

follow-up digital subtraction angiography examination after 6 months confirmed the good result of the procedure (Figure $1 \mathrm{D}$ ).

There are some technical issues associated with ET of such challenging cases that should be discussed. Implantation of stents in the intracranial segments of the ICA is associated with a high rate of severe complications, at the level of $5-15 \%$. Therefore, the use of DEBs seems to be a promising alternative $[3,4]$. There is also a high risk of periprocedural peripheral embolization; thus the use of proximal protection devices, which shield the brain during the procedure and allow for the use of any guidewire, seems indispensable. There are also some advantages of the Elutax " 3 " Neuro balloon. This device is dedicated to the treatment of lesions in the IA. It can also be used without prior predilation, which reduces the risk of dissection and the need for stent implantation [4]. Regarding postprocedural pharmacotherapy after the use of stents or DEB in IA, no widely accepted recommendations exist at the moment. In our patients we routinely use DAPT for 6-12 months. In this case, we asked the patient to take DAPT for 6 months, until the follow-up; then, he received only aspirin.

Finally, it should be emphasized that although ET of symptomatic stenosis of intracranial segments of the ICA can be a life-saving procedure, it should be performed exclusively in centers with high expertise in carotid interventions.

\section{Conflict of interest}

The authors declare no conflict of interest.

\section{References}

1. Vellimana AK, Ford AL, Lee JM, et al. Symptomatic intracranial arterial disease: incidence, natural history, diagnosis, and management. Neurosurg Focus 2011; 30: e14.

2. Gruber P, Garcia-Esperon C, Berberat J, et al. Neuro Elutax SV drug-eluting balloon versus Wingspan stent system in symptomatic intracranial high-grade stenosis: a single-center experience. J Neurointerv Surg 2018; 10: e32.

3. Gruber P, Remonda L. Device profile of different paclitaxel-coated balloons: Neuro Elutax SV, Elutax '3' Neuro and SeQuent Please NEO for the treatment of symptomatic intracranial highgrade stenosis: overview of their feasibility and safety. Expert Rev Med Devices 2020; 17: 87-92.

4. Soler EE, Serrano BS, Hernández NL, et al. Transcranial duplex ultrasound monitoring of intracranial arterial stenosis treated with ELUTAX "3" drug-eluting balloon. Interv Neuroradiol 2020; 26: 800-4. 